AperTO - Archivio Istituzionale Open Access dell'Università di Torino

\title{
Dermal-Epidermal Cross-Talk: Differential Interactions With Microvascular Endothelial Cells
}

\section{This is a pre print version of the following article:}

Original Citation:

Availability:

This version is available http://hdl.handle.net/2318/1612278

since 2017-05-12T19:00:14Z

Published version:

DOI: $10.1002 / j c p .25657$

Terms of use:

Open Access

Anyone can freely access the full text of works made available as "Open Access". Works made available under a Creative Commons license can be used according to the terms and conditions of said license. Use of all other works requires consent of the right holder (author or publisher) if not exempted from copyright protection by the applicable law. 
This is the author's final version of the contribution published as:

Bassino, Eleonora; Vallariello, Edoardo; Gasparri, Franco; Munaron, Luca. Dermal-Epidermal Cross-Talk: Differential Interactions With Microvascular Endothelial Cells. JOURNAL OF CELLULAR PHYSIOLOGY. None pp: ---. DOI: $10.1002 /$ jcp. 25657

The publisher's version is available at:

http://onlinelibrary.wiley.com/wol1/doi/10.1002/jcp.25657/fullpdf

When citing, please refer to the published version.

Link to this full text:

http://hdl.handle.net/ 
FROM THE BENCH

DERMAL-EPIDERMAL CROSS-TALK: DIFFERENTIAL INTERACTIONS WITH MICROVASCULAR ENDOTHELIAL CELLS.

Eleonora Bassino ${ }^{1}$ Edoardo Vallariello ${ }^{1}$, Franco Gasparri $^{2}$ \& Luca Munaron $^{1 *}$

${ }^{1}$ Deparment of Life Sciences and Systems Biology, University of Turin, Italy. ${ }^{2}$ Department of Pharmacy, University of Salerno, Italy.

*Correspondence to:

Luca Munaron, Ph.D.

Dept. Life Sciences \& Systems Biology

University of Torino

Via Accademia Albertina 13

10123 Torino

ITALY

luca.munaron@unito.it

Key words: endothelial cells, hair follicle, coculture, keratinocytes, angiogenesis.

Running Title: Paracrine cross-talk between dermal-epidermal and endothelial layers.

Total number of text figures and tables: 3 Figures; 0 Tables. 


\begin{abstract}
The biological importance of circulatory blood supply and angiogenesis for hair growth is now well recognized, but the their regulatory mechanisms require more mechanistic investigation. In vitro cocultures and tricultures can be successfully employed to greatly improve our knowledge on paracrine crosstalk between cell types that populate the dermal-epidermal interface and cutaneous vasculature. Here we report that human dermal fibroblasts (NHDF) promote viability and proliferation of microvascular endothelial cells (HMVEC), while HMVEC are not mitogenic for NHDF. In triculture setup, conditioned media obtained by cocultures (HMVEC/NHDF or HMVEC/follicle fibroblasts) differently modulate growth and proliferation of keratinocytes and alter the expression of metabolic and pro-inflammatory markers. In conclusion, tricultures were successfully employed to characterize in vitro dermal-epithelial and endothelial interactions and could integrate ex vivo and in vivo approaches by the use of high-throughput and standardized protocols in controlled conditions.
\end{abstract}




\section{INTRODUCTION}

Human cutaneous vasculature $(\mathrm{CV})$ is organized in two major plexuses of blood vessels and capillaries can also be found within dermal papilla of hair follicle (HF) structures (Yano et al., 2001). In the adult, skin angiogenesis is still thought to be restricted to pathological conditions such as wound healing (Tonnesen et al., 2000), hyperproliferative inflammatory skin diseases like psoriasis (Varricchi et al., 2015), and in association with a wide range of tumors (Stapor et al., 2014). Physiological development and cycling of HF depends on the interaction between epithelial and mesenchymal cells (Schmidt-Ullrich et al., 2005), being able to develop and grow without a direct blood supply, as revealed by different organ culture techniques (Hardy et al., 1992; Philpott et al., 1999). However, blood supply is essential for long term hair maintenance, bringing nutrients, growth factors, cytokines and other bioactive molecules. In particular, vascular endothelial growth factor (VEGF), a key player in angiogenesis and vascular permeability, controls hair growth and follicle size (Yano et al., 2001). Conversely, in vitro and ex vivo evidences report that cytokines such as IL- $1 \alpha$ are negative regulators of hair cycle (Hamada et al., 2003; Boivin et al., 2006; Harmon et al., 1993).

The intensive circulatory support during anagen suggests its association with the high metabolic activity of HF matrix cells and insufficient blood supply can lead to HF diseases (Schmidt-Ullrich et al., 2005). Moreover, anagen HF, similarly to interfollicular epidermis, display angiogenic properties in vivo (Schmidt-Ullrich et al., 2005). Several techniques have been developed to characterize HF reconstitution and skin wound repair in more detail, both in vivo and in vitro.

The hair inductive properties of dermal cells were first shown by Oliver et al., (1967) in which DP were transposed beneath the upper half of amputated vibrissa HF. Recently, two hair regeneration models were established using dissociated cell aggregates or single cells from HF as the epidermal and dermal components. The first model is based on the use of a silicon graft chamber, in which a mixture of epidermal and dermal cells is placed onto a full thickness wound on the back of 
immunodeficient mice (Lichti et al., 2008). In the second application, the same mixture is directly injected subcutaneously into the back skin of immunodeficient mice (Morris et al., 2004). In vivo animal models preserve the complex, spatially organized environment including systemic interactions: however, they suffer for a limited control and knowledge of molecular dynamics underlying paracrine mechanisms. These systems are supported by in vitro methodology that includes the use of collagen as a matrix for dermal-epidermal interaction. Epidermal and dermal cells can either be incorporated into the gel or sit on the top of the gel (Zheng et al., 2005). Dermalepidermal interaction can also take place in a hanging-drop system (Qiao et al., 2008). Wound healing process can be also investigated by in vitro approaches (Ehrlich et al., 2003). Here we show that cell cocultures and tricultures represent a promising in vitro tool to shed light on the molecular mechanisms responsible for the paracrine and functional interaction between dermal components and keratinocytes in strictly controlled and reproducible conditions. 


\section{MATERIALS AND METHODS}

\section{Cell Cultures}

- Human hair follicle dermal papilla cell line (FDPC) was obtained from PromoCell (Germany; lot 2040301.35). FDPC were grown in Follicle Dermal Papilla Cell Medium (PromoCell) with 1\% antibiotic/antimycotic (Invitrogen, Grand Island, NY, USA). In order to avoid phenotypical changes occurring during cell culture, only FDPC at 2-7 passages were used for all experiments. To confirm FDPC stability we measured alkaline phosphatase activity (ALP). ALP activity: (U/ml)=A/V/T, 1.62 (Bassino et al., 2015).

- Human Adult Keratinocyte cell line (HaCaT) was obtained from Cell Line Services (CLS Cell Lines Service, Germany). HaCaT were grown in Dulbecco’s Modified Eagle medium (DMEM) with 10\% FCS and 1\% antibiotic/antimycotic (Invitrogen, Grand Island, NY, USA).

- Human microvascular endothelial cell line (HMVEC) was purchased from Lonza (Human adult dermal microvascular endothelial cells, include cells isolated from small vessels within skin tissue from either adults or neonatal foreskins) and grown in Endothelial Cell Grown Medium (EGM 2-MV medium, Lonza).

- Normal Human Dermal Fibroblast (NHDF) was purchased from Lonza and grown in FGM $^{\text {TM}}-2$ Fibroblast Growth Medium-2 (Lonza) that contains 2\% serum and $1 \%$ antibiotic/antimycotic (Invitrogen, Grand Island, NY, USA).

All cell types were maintained at $37^{\circ} \mathrm{C}$ in a humidified atmosphere of $5 \% \mathrm{CO}_{2}$.

\section{Cocultures}

HMVEC were thawed from frozen stock and seeded in 24 well plates at a density of $3 \times 10^{3}$ (for cell viability assays) or $2.5 \times 10^{3}$ cells/well (for proliferation assays). 
For cell viability in coculture, FDPC or NHDF were thawed, centrifuged, recounted and seeded at a density of $3 \times 10^{3}$ cells/well for 2 days in Dulbecco's Modified Eagle Medium (DMEM) 10\% Fetal Calf Serum (FCS) until 100\% confluence on transwell clear polyester membrane inserts with 0.4$\mu \mathrm{m}$ pore size and a $0.3-\mathrm{cm}^{2}$ area (Corning).

\section{Tricultures}

To realize tricultures, HaCaT were seeded in 24 or 96 well plates for 2 days in DMEM 10\% FCS; then DMEM 10\% was substituted with DMEM 0, $10 \%$ or with conditioned media (CM) obtained from cocultures.

\section{Cell counting assays}

Cell Viability. In viability assays in cocultures, HMVEC were plated in 24 well plates at the density of $5 \times 10^{3}$ (near confluence) NHDF were thawed, centrifuged, recounted and seeded at a density of $3 \times 10^{3}$ cells/well for 2 days in Dulbecco's Modified Eagle Medium (DMEM) $+10 \%$ Fetal Calf Serum (FCS) until 100\% confluence on Transwell clear polyester membrane inserts with $0.4-\mu \mathrm{m}$ pore size and a $0.3-\mathrm{cm}^{2}$ area (Corning). The viability of HMVEC (alone or in the presence of NHDF or medium alone on the insert) was analyzed at 24 hrs. For viability assays in tricultures, cells were plated in 24 well plates at the density of $5 \times 10^{3}$. Cell viability was analysed after $24 \mathrm{hrs}$ of incubation with DMEM 0,10\%, or CM (FDPC-HMVEC: CM1 or NHDF-HMVEC: CM2). As described above for NHDF, HMVEC were seeded on the transwell clear polyester membrane inserts and exposed to the same experimental protocol for survival assays.

Cell Proliferation. In proliferation assays in co-cultures, HMVEC were seeded at a density of $2.5 \mathrm{x}$ $10^{3}$ cells/well (lower than in 'cell viability' assays), medium was discarded after $18 \mathrm{hrs,} \mathrm{then}$ complete medium was replaced with DMEM 2\% FCS to reduce cell proliferation for 24 hrs and finally replaced with DMEM $10 \%$ or $0 \%$ in the presence of the insert with or without NHDF. Cell number was evaluated after $48 \mathrm{hrs}$. As described above for NHDF, HMVEC were seeded on the 
transwell clear polyester membrane inserts and exposed to the same experimental protocol for proliferation assays. For proliferation in tricultures, HaCaT were seeded at a density of $2.5 \times 10^{3}$ cells/well, medium was discarded after $18 \mathrm{hrs,} \mathrm{then} \mathrm{complete} \mathrm{medium} \mathrm{was} \mathrm{replaced} \mathrm{with} \mathrm{DMEM}$ $2 \%$ FCS to reduce cell proliferation for $24 \mathrm{hrs}$ and finally replaced with DMEM $10 \%$ or $0 \%$ in the presence CM obtained from cocultures (CM1: FDPC/HMVEC or CM2: NHDFHMVEC). Cell number was evaluated after $48 \mathrm{hrs}$.

Cell viability ( $24 \mathrm{hrs}$ ) and proliferation ( $48 \mathrm{hrs}$ ) were evaluated by the CellTiter $96 \AA$ AQueous NonRadioactive Cell Proliferation Assay (Promega, Madison, WI, USA), using 3-(4,5-dimethylthiazol2-yl)-5-(3-carboxymethoxyphenyl)-2-(4-sulfophenyl)-2H tetrazolium, inner salt (MTS). MTS conversion into the aqueous soluble formazan product is accomplished by dehydrogenase enzymes found in metabolically active cells. Formazan product was measured with a FilterMax F5 Microplate reader (Molecular Devices, US) at $490 \mathrm{~nm}$, as absorbance is directly proportional to the number of viable cells.

\section{Enzyme-linked immunosorbent assays (ELISA)}

$\beta$-catenin, EGF and IL-1 $\alpha$ levels (in cell lysates or medium) were assessed by enzyme-linked immunosorbent assay (ELISA) using commercially available kits (Sigma ELISA kit, Invitrogen ELISA Kit, respectively). Briefly, $100 \mu \mathrm{L}$ of medium (EGF or IL-1 $\alpha$ ) or cell lysate (nuclear cell lysate for $\beta$-catenin; See below 'Nuclear protein extraction for $\beta$-catenin quantification') were incubated into an antibody-coated 96 -well plate at room temperature for 2,5 hrs. The wells were washed four times with wash buffer solution. Then $100 \mu \mathrm{L}$ anti-human $\beta$-catenin, EGF or IL$1 \alpha$ antibody was added and the samples were again incubated for $1 \mathrm{hr}$ at room temperature. The plate was washed four times, $100 \mu \mathrm{L}$ of streptavidin-peroxidase conjugated was applied for $1 \mathrm{hr}$ at room temperature. After a final washing, $100 \mu \mathrm{L}$ tetramethylbenzidine substrate was added and allowed to develop for $30^{\prime}$ in the dark at room temperature. After stopping the reaction with $50 \mu \mathrm{L}$ stop solution containing citric acid $2.0 \mathrm{mmolL}^{-1}$, absorbance was read at $450 \mathrm{~nm}$ with a F5 
FilterMax microplate reader 550 (Molecular Devices, US). Sample concentration was calculated from the standard curve.

\section{Nuclear protein extraction for $\beta$-catenin quantification}

HaCaT were grown to $70-80 \%$ confluence. Afterwards, cells were scraped using fresh PBS, collected into an appropriate conical tube and centrifuged ( $5^{\prime}$ at $450 \mathrm{x} g$ ). Then the supernatant was discarded and $1 \mathrm{~mL}$ Lysis Buffer $(10 \mathrm{mM}$ Tris $\mathrm{HCl}, \mathrm{pH} 7.5,2 \mathrm{mM} \mathrm{MgCl}, 3 \mathrm{mM} \mathrm{CaCl}, 0.3 \mathrm{M}$ Sucrose, including DTT and Protease Inhibitors) added to $200 \mu \mathrm{L}$ of packed cell volume (PCV) for 15 minutes. Suspended cells were centrifuged for 5 minutes at 420 x $g$. Pellet of packed cells was resuspended in $400 \mu \mathrm{L}(2 \mathrm{X}$ PCV) Lysis Buffer and fragmented using a syringe with a narrowgauge. The disrupted cells in suspension were centrifuged for $20 \mathrm{~min}$ at $10,000 \mathrm{x} g$. The supernatant was transferred into a fresh tube and this fraction corresponds to the cytoplasmic fraction. Subsequently the pellet was resuspended in $140 \mu$ l extraction buffer (20 mM HEPES, pH 7.9, with $1.5 \mathrm{mM} \mathrm{MgCl}_{2}, 0.42 \mathrm{M} \mathrm{NaCl}, 0.2 \mathrm{mM}$ EDTA, $25 \%$ (v/v) Glycerol added with $1.5 \mu \mathrm{L}$ of the $0.1 \mathrm{M}$ DTT solution and $1.5 \mu \mathrm{L}$ of the Protease Inhibitor Cocktail) and centrifuged for 5 minutes at 20,000 $\mathrm{x} g$. The resulting supernatant is the nuclear protein extract. It is finally collected to a clean tube and analyzed with $\beta$-catenin ELISA assay.

\section{Lipid peroxidation}

Lipid peroxidation was analysed using Click-iT® LAA reaction kit (LifeTechologies). Briefly, Linoleic acid is the most abundant polyunsaturated fatty acid found in mammals and its lipid peroxidation products likely account for the majority of lipid-derived protein carbonyls. When incubated with cells, LAA (Linoleamide alkyne) incorporates into cellular membranes. Upon lipid peroxidation, LAA is oxidized and produces 9- and 13-hydroperoxy-octadecadienoic acid (HPODE). These hydroperoxides decompose to multiple $\alpha, \beta$-unsaturated aldehydes, which readily modify proteins at nucleophilic side chains. These alkyne-containing modified proteins can be 
subsequently detected using Click-iT® chemistry and multiplexed with other probes appropriate for fixed cells. Fluorescence was read with a F5 FilterMax microplate reader 550 (Molecular Devices, US) and observed with fluorescence microscope (Nikon T-E microscope, 10x objective).

\section{Fluorescence microscopy: Keratin-1 and -81 determination}

Keratin-1 and -81 (KRT1 or KRT81) were detected using immunofluorescence assay. Briefly, after the treatments cells were fixed in 4\% paraformaldehyde for 25 minutes at room temperature. Then the fixed cells were washed three times with ice cold PBS solution, incubated $20^{\prime}$ with $0.3 \%$ Triton and $1 \%$ bovine serum albumin (BSA, Sigma) in PBS, and stained with the primary antibody 24 hrs at $4^{\circ} \mathrm{C}$. Cover slides were washed twice with PBS and incubated $1 \mathrm{hr}$ at room temperature with the secondary antibody. After two washes in PBS, cover slides were mounted on standard slides with DABCO (Sigma) and observed after $24 \mathrm{hrs}$ with by fluorescence microscopy (Nikon T-E microscope, 10x objective). For each experiment we randomly acquired three fields/sample evaluating the mean of fluorescence intensity/cell/field (about 30 cells/field).

\section{Scratch wound healing}

HaCaT were seeded in 24 multi-well plates and culture until confluent. A scratch was made in the confluent monolayer with a plastic disposable pipette tip $(100 \mu 1)$. Debris was removed from the culture by gently washing with sterile PBS. Hereafter, HaCaT were cultured in DMEM 10\%, DMEM 0\%, CM1 or CM2 media for 24 hrs. Experiments were performed using a Nikon T-E microscope (4x objective). Cells were kept at $37{ }^{\circ} \mathrm{C}$ and $5 \% \mathrm{CO}_{2}$ for all experiments; Photos were taken every 4 hrs using Metamorph software. Cell migration was measured with ImageJ software. At least three fields for each condition were analyzed in each independent experiment.

\section{Statistical analysis}

Statistical significance of all experiments was evaluated by Kaleidagraph software (Synergy 
Software, USA) using nonparametric Wilcoxon test. All values are presented as the mean \pm standard error $(\mathrm{SEM}) . \mathrm{N}=5$ technical replicates were performed for each experimental condition; $\mathrm{N}=3$ biological replicates were performed for each experimental condition. Results with $p$-values $<0.05$ were considered statistically significant: ${ }^{*} \mathrm{p}<0.05 ; * * \mathrm{p}<0.01 ; * * * \mathrm{p}<0.001$. 


\section{RESULTS}

\section{NHDF promote HMVEC survival and proliferation}

Recently, we demonstrated that the viability of starved HMVEC was significantly enhanced when cocultured in presence of NHDF (24 hrs) (Bassino et al., 2015). In the present work we observed that NHDF were also able to stimulate HMVEC proliferation upon $48 \mathrm{hrs}$ of coculture (Fig. 1A). In a complementary coculture configuration, HMVEC seeded in the insert failed to sustain viability (Fig. 1B, $24 \mathrm{hrs)}$ and proliferation of NHDF (Fig. 1C, $48 \mathrm{hrs).}$

\section{Conditioned media modulate survival and proliferation of keratinocytes}

The role of conditioned media obtained by FDPC-HMVEC or NHDF-HMVEC cocultures (CM1 or $\mathrm{CM} 2$, respectively) was tested on human keratinocytes. HaCaT viability was maximal when cells were grown in complete medium (DMEM 10\% FCS) and was drastically decreased upon serum deprivation (DMEM 0\% FCS, $24 \mathrm{hrs)} \mathrm{(Fig.} \mathrm{2A,} \mathrm{See} \mathrm{schematic} \mathrm{configurations} \mathrm{1-3).} \mathrm{Incubation} \mathrm{of}$ HaCaT with both CMs significantly promoted cell survival (Fig. 2A). This effect was more prominent for CM1 that exerted also a significant mitogenic activity for HaCaT, whereas CM2 resulted ineffective (48 hrs Fig. 2B, See schematic configurations 1-3).

\section{Modulation of wound closure and anti-inflammatory role of conditioned media}

To evaluate the role of $\mathrm{CMs}$ in the wound healing rate of $\mathrm{HaCaT}$, we first examined their ability to modulate cell motility employing an established in vitro scratch wound healing assay. Wound closure was evaluated by observing the re-populated area between the wound margins at different time intervals (0-, 8- $24 \mathrm{hrs})$ after the lesion. The wound monitoring showed that untreated cells (DMEM 10\%) followed the physiological healing process, reaching approximately $45 \%$ of closure 24 hrs after injury (Fig. 2C, See schemes 4-6). Both CM1 and CM2 were able to enhance healing process reaching $70 \%$ and $75 \%$ of closure, respectively, 24 hrs after injury (Fig. 2D). 
Skin wounding and inflammatory responses involve Interleukin-1 $\alpha$ (IL-1 $\alpha$ ), a cytokine that exerts inhibitory activity on human hair follicle growth and its cycle, not only during anagen-catagen switch, but also in anagen induction (Harmon et al., 1993; Hamada et al., 2003). We investigated the effects of CM1 on cytokine production by keratinocytes treated with $\mathrm{H}_{2} \mathrm{O}_{2}(500 \mu \mathrm{M}, 24 \mathrm{hrs})$, a strong oxidative stress stimulus. As expected, $\mathrm{H}_{2} \mathrm{O}_{2}$ drastically enhanced IL- $1 \alpha$ levels in HaCaT, an effect significantly prevented by CM1 incubation (Fig. 3A). Wounded keratinocyte monolayers expressed high levels of IL-1 $\alpha$ when treated with $\mathrm{H}_{2} \mathrm{O}_{2}$ and $\mathrm{CM} 1$ failed to reduce cytokine production (Fig. 3A). Recently, we reported that human fibroblasts completely fail to affect IL-1 $\alpha$ release triggered by oxidative stress on HMVEC treated with $\mathrm{H}_{2} \mathrm{O}_{2}(400 \mu \mathrm{M}, 2 \mathrm{hrs})$ (Bassino et al., 2015). Here, we analyzed the potential anti-inflammatory role of CM1 and CM2 measuring lipid oxidation by $\mathrm{HaCaT}$ treated with $\mathrm{H}_{2} \mathrm{O}_{2}(500 \mu \mathrm{M}, 24 \mathrm{hrs})$; in this condition both CM significantly reduced lipid peroxidation in $\mathrm{HaCaT}$ exposed to oxidative stress (Fig. 3B, See schemes 1-4).

\section{Expression of metabolic markers}

Both CM1 and CM2 enhanced the expression of nuclear $\beta$-catenin, a key player in the regulation of follicle cell cycle, cell adhesion and signaling (Fagotto 2013), by HaCaT upon short-term treatment (24 hrs, Fig. 3C, Schemes 1-4). A similar activity was observed for both CM1 and CM2 on the release of the growth factor EGF from keratinocytes. Interestingly, these responses were induced both on 'healthy' conditions and upon mechanical damage (wounded HaCaT monolayer) (Fig. 3D, Schemes 1-4).

Afterwards, we investigated the expression of hair cytokeratins (KRT81 and KRT1). KRT81 is a marker for keratinocytes that differentiate in hair or nails (Langbein et al., 2005), KRT1 is a constitutive cytokeratin usually expressed in the spinous and granular layers of the epidermis (Langbein et al., 2005). Fluorescence microscopy evidenced that HaCaT express cytosolic basal levels of both KRT81 and KRT1 under physiological conditions (DMEM 10\%), showing a decrease upon serum starvation (DMEM 0\%). CM1 significantly increased KRT81 expression after 24 hrs of 
treatment (Fig. 3E), while CM2 reduced KRT1 levels (Fig. 3F). 


\section{DISCUSSION}

Several experimental strategies have been developed to investigate HF reconstitution and skin wound repair. The use of in vivo approaches, that retain the complex microenvironment and tissue organization, evidenced that the inhibition of KGF receptor signalling in basal keratinocytes of transgenic mice causes a severe delay in re-epithelialization (Werner et al., 1994). Recently, Heidari et al., (2016) reported the ability of HF stem cells to accelerate cutaneous wound healing in rats. However, these reports cannot enter into molecular and mechanistic details of these events, due to the high complexity of the environment. On the other hand, in vitro protocols provide highly simplified but more controlled conditions: among them, cocultures allow different cell populations to grow interacting each other in different extents. A synergistic mix of growth factors, extracellular matrix components, and direct or indirect cell-cell contacts, supports epidermal stem cell phenotype and keratinocyte proliferation (Nelson et al., 2006). Interestingly, fibroblasts in collagen gels form gap junctions, which are also involved in the regulation of extracellular matrix production (Ehrlich et al., 2006). Recently, we used insert-based cocoltures to showe that HF fibroblasts promote survival, proliferation and tubulogenesis of microvascular endothelial cells more efficiently than common dermal fibroblasts, collectively suggesting a key role of papilla cells in vascular remodeling of the HF (Bassino et al., 2015). Here we implemented that approach, conditioning keratinocites with the medium obtained by cocultures of endothelial cells with follicle dermal papilla cells (FDPC) or interfollicle dermal fibroblasts (NHDF) (CM1 or CM2, respectively). We report that CM1 strongly enhances viability and proliferation of keratinocytes; conversely, CM2 slightly increases cell survival but it is not mitogenic. Either conditioned media significantly enhance keratinocyte wound healing ( $24 \mathrm{hrs}$ ). The ability of hair follicles to repair chronic cutaneous wound sites is reported in literature (Liu et al., 2015), but it is interesting to notice that in our study inter-follicular NHDF increase wound repair similarly to FDPC. Moreover, CMs differentially alter the expression of $\beta$-catenin and the release of EGF. $\beta$-Catenin regulates 
keratinocyte stem cell differentiation, hair follicle morphogenesis, and protects the epidermis from mechanical stresses (Ray et al., 2013); on the other hand, EGFR pathway has a major impact on the inflammatory/immune reactions of the skin protecting from the overactivation of keratinocyte proinflammatory functions (Pastore et al., 2008). Treatment with CMs also triggers differential expression of cytokeratines (KRT1 and KRT81), that could be related to keratinocyte maturation. Indeed, expression of KRT1 can be viewed as a differentiation marker, as suggested by the evidence that some basal keratinocytes specialize, lose their mitotic activity and start to express KRT1 which form the intracellular keratin network, together with keratin-binding proteins (Langbein et al., 2005). Similarly, KRT81 is expressed by specific keratinocytes that differentiate in hair or nails (Langbein et al., 2005). Moreover, in FDPC/HMVEC cocultures, we recently reported that the proangiogenic growth factors VEGF and IGF-I were released by both cell types; in particular, FDPC produced more VEGF than the endothelium (Bassino et al., 2015). Therefore, we hypothesize that these growth factors, in association with other unidentified molecules released by cocultures, could be involved in the paracrine modulation of keratinocyte functions. In conclusion, tricultures setted in this work are a suitable tool aimed to extend our view of the complex relationship between dermal, epithelial and endothelial layers. A relevant conclusion is that the coculture of microvascular endothelium with specialized fibroblasts (FDPC) is more powerful than the association with common dermal fibroblasts.

We acknowledge the limitations of in vitro setups that take into account only a fraction of the complex architecture in native organs: nonetheless, these methods could successfully integrate and support the well-established ex vivo and in vivo approaches, in particular for the controlled and standardized screening of new promising drugs with dermatological applications as well as of the molecular pathways that mediate their functional effects.

Conflicts of interest: None declared

\section{Acknowledgements}


E.B. designed the research study, performed the research, analysed the data and wrote the paper E.V. performed some experiments

F.G. contributed essential reagents or tools and designed the research study

L.M. designed the research study, analysed the data, wrote the paper and contributed essential reagents or tools. 


\section{REFERENCES}

Bassino E, Gasparri F, Giannini V, Munaron L. 2015. Paracrine crosstalk between human hair follicle dermal papilla cells and microvascular endothelial cells. Experimental Dermatology 24: $388-390$.

Boivin WA, Jiang H, Utting OB, Hunt DW. 2006. Influence of interleukin-1alpha on androgen receptor expression and cytokine secretion by cultured human dermal papilla cells. Exp Dermatol. 15:784-93.

Ehrlich HP, Diez T. 2003. Role for gap junctional intercellular communications in wound repair. Wound Repair Regen 11:481-9.

Ehrlich HP, Sun B, Saggers GC, Kromath F. 2006. Gap junction communications influence upon fibroblast synthesis of Type I collagen and fibronectin. J Cell Biochem. 98:735-43.

Fagotto F. 2013. Looking beyond the Wnt pathway for the deep nature of $\beta$-catenin. EMBO Reports. 14:422-33.

Hamada K, Hirotsu S, Uchiwa H, Yamazaki S, Suzuki K. 2003. Pro-inflammatory cytokine interleukin- $1 \alpha$ is downregulated during anagen phase of hair cycle in vivo. Journal of dermatological Science. 33:195-198.

Hardy MH. 1992. The secret life of the hair follicle. Trends Genet 8: 55-61.

Harmon CS, Nevins T. 1993. IL-1 alpha inhibits human hair follicle growth and hair fiber production in whole-organ cultures. Lymphokine Cytokine Res 12:197-203.

Heidari F, Yari A, Rasoolijazi H, Soleimani M, Dehpoor A, Sajedi N, Joulai Veijouye S, N. M. 2016. Bulge Hair Follicle Stem Cells Accelerate Cutaneous Wound Healing in Ra. Wounds 28 : $132-41$.

Langbein L, Schweizer J. 2005. Keratins of the Human Hair Follicle. International Review of Cytology 243:1-78.

Lichti U, Anders JY S. 2008. Isolation and short-term culture of primary keratinocytes, hair follicle populations and dermal cells from newborn mice and keratinocytes from adult mice for in vitro analysis and for grafting to immunodeficient mice. Nat Protoc 3: 799-810. 
Liu JQ, Zhao KB, Feng ZH, Qi FZ. 2015. Hair follicle units promote re-epithelialization in chronic cutaneous wounds: A clinical case series study. Experimental and Therapeutic Medicine 10: $25-30$.

Morris RJ Liu Y, Marles L, Yang Z, Trempus C, Li S, Lin JS, Sawicki JA, Cotsarelis G. 2004. Capturing and profiling adult hair follicle stem cells. Nat Biotechnol 22:411-417.

Nelson CM, Bissell MJ. 2006. Of extracellular matrix, scaffolds, and signaling: tissue architecture regulates development, homeostasis, and cancer. Annu Rev Cell Dev Biol 22:287-309.

Oliver RF. 1967. The experimental induction of whisker growth in the hooded rat by implantation of dermal papillae. J Embryol Exp Morphol 18: 43-51.

Pastore S, Mascia F, Mariani V, Girolomoni G. 2008. The Epidermal Growth Factor Receptor System in Skin Repair and inflammation. Journal of Investigative Dermatology 128:13651374.

Philpott M. 1999. In vitro maintenance of isolated hair follicles: current status and future development. Exp Dermatol 8:317-9.

Qiao J, Turetsky A, Kemp P, Teumer J. 2008. Hair morphogenesis in vitro: formation of hair structures suitable for implantation. Regenerative Medicine 3: 683-692.

Ray S, Foote HP, Lechler T. 2013. $\beta$-Catenin protects the epidermis from mechanical stresses. J. Cell Biol. 202: 1 45-52.

Schmidt-Ullrich R, Paus R. 2005. Molecular principles of hair follicle induction and morphogenesis. Bioessays 27:247-261.

Stapor P, Wang X, Goveia J, Moens S, Carmeliet P. 2014. Angiogenesis revisited - role and therapeutic potential of targeting endothelial metabolism. J Cell Sci. 27:4331-41.

Tonnesen MG, Feng X, Clark RA. 2000. Angiogenesis in Wound Healing. Journal of Investigative Dermatology Symposium Proceedings 51: 40-46.

Varricchi G, Granata F, Loffredo S, Genovese A, Marone G. 2015. Angiogenesis and lymphangiogenesis in inflammatory skin disorders. J Am Acad Dermatol. 73:144-53.

Werner S, Smola H, Liao X, Longaker MT, Krieg T, Hofschneider PH, Williams LT. 1994. The function of KGF in morphogenesis of epithelium and reepithelialization of wounds. Science (New York, N.Y.) 266:, 819-22. 
Yano K, Brown LF, Detmar M. 2001. Control of hair growth and follicle size by VEGF-mediated angiogenesis. J Clin Invest. 107:409-17.

Zheng Y, Du X, Wang W, Boucher M, Parimoo S, Stenn KS. 2005. Organogenesis from dissociated cells: Generation of mature cycling hair follicles from skin-derived cells. Journal of Investigative Dermatology 124: 867-876. 


\section{FIGURE LEGENDS}

\section{Figure 1. Non reciprocal functional interaction between NHDF and HMVEC.}

Scheme of the experimental setup. Arabic numbers in graph (1-3) are referred to the monoculture and coculture configurations.
A. NHDF increased HMVEC number in cell proliferation assays (48 hrs).
B. HMVEC failed to sustain cell viability of starved NHDF in a complementary coculture configuration (24 hrs).
C. HMVEC failed to sustain cell proliferation of NHDF (48 hrs).
Color coding: red: cocultures; black: monocultures.

Figure 2. Effects of conditioned media on viability, proliferation and wound healing of HaCaT.

Scheme of the experimental setup. Arabic numbers in graphs (1-6) are referred to monocultures, cocultures and tricultures.

A. CM1 and CM2 significantly increased $\mathrm{HaCaT}$ viability (24 hrs). In coculture configuration only FDPC significantly increased HaCaT survival (I). No change in HaCaT viability cocultured with HMVEC or NHDF (II, III).

B. CM1 increased HaCaT proliferation (48 hrs). No change was observed in the presence of CM2.

C. Both CMs promoted wound healing closure. Representative microphotographs of wound healing: untreated and CM-treated HaCaT monolayers at 0,8 and 24 hrs after injury (10X magnification).

D. Wound healing quantification. The closure percentage values are referred to $24 \mathrm{hrs}$ of treatment.

Color coding: red: tricultures; green: cocultures; black: monocultures.

Figure 3. Effects of conditioned media on keratinocyte inflammatory response and expression of different markers.

Scheme of the experimental setup. Arabic numbers in graph (1-4) are referred to the monoculture, coculture and triculture configurations.

A. IL-1 $\alpha$ quantification on confluent or wounded $\mathrm{HaCaT}$ treated with $\mathrm{H}_{2} \mathrm{O}_{2}$. CM1 significantly reduced IL-1 $\alpha$ production on confluent $\mathrm{HaCaT}$ but was unable to affect wounded HaCaT.

B. In lipid peroxidation assays performed on confluent $\mathrm{HaCaT}$ treated with $\mathrm{H}_{2} \mathrm{O}_{2}$, both $\mathrm{CM} 1$ and CM2 significantly reduced oxidative stress $\left(\mathrm{B}_{\mathrm{i}}\right)$; representative fluorescence images of lipid peroxidation $\left(\mathrm{B}_{\mathrm{ii}}\right)$. 
C. CM1 affects nuclear $\beta$-catenin expression on both confluent and wounded HaCaT ( $24 \mathrm{hrs})$.

D. EGF levels were increased by both CM1 and CM2 on confluent HaCaT, while only CM1 significantly increased EGF release by wounded HaCaT (24 hrs).

E. Immunofluorescence experiments: CM1 increased KRT81 fluorescence intensity (F.I.) in $\mathrm{HaCaT}(24 \mathrm{hrs})$.

F. Immunofluorescence experiments: CM2 decreased KRT1 fluorescence intensity (F.I.) in HaCaT (24 hrs).

Color coding: red: wound condition. 